\title{
Potential astrophysics science missions enabled by NASA's planned Ares V
}

\author{
H. Philip Stahl \\ NASA Marshall Space Flight Center, Huntsville, AL 35812 \\ Harley Thronson \\ NASA Goddard Space Flight Center, Greenbelt, MD \\ Stephani Langhoff \\ NASA Ames Research Center, Mountain View, CA \\ Marc Postman \\ Space Telescope Science Institute, Baltimore, MD \\ Daniel Lester \\ University of Texas, Austin, TX \\ Chuck Lillie \\ Northrop Grumman Corporation, Los Angeles, CA
}

\begin{abstract}
NASA's planned Ares V cargo vehicle with its 10 meter diameter fairing and $\sim 60,000 \mathrm{~kg}$ payload mass to $\mathrm{L} 2$ offers the potential to launch entirely new classes of space science missions such as 8-meter monolithic aperture telescopes, 12meter aperture x-ray telescopes, 16 to 24 meter segmented telescopes and highly capable outer planet missions. The paper will summarize the current Ares V baseline performance capabilities and review potential mission concepts enabled by these capabilities.
\end{abstract}

Keywords: Astronomy, Large Space Telescopes, Ares V Launch Vehicle

\section{INTRODUCTION}

NASA's Ares V cargo launch vehicle is a disruptive capability whose payload mass and volume capacities offer the potential to completely change the paradigm of future space science mission. Future space science telescopes desire increasingly larger telescope collecting aperture. But, current launch vehicle mass and volume constraints are a severe limit. The Ares $\mathrm{V}$ greatly relaxes these constraints. For example, while current launch vehicles have the ability to launch approximate a 4.5 meter diameter payload with a mass of 13,000 kg to Earth-Sun L2, the Ares V is projected to have the ability to launch an 8.8 meter diameter payload with a mass of $\sim 60,000 \mathrm{~kg}$ to L2 or a mass of $\sim 180,000 \mathrm{~kg}$ to Low Earth Orbit. The Ares V mass and volume payload capabilities have the potential to enable entirely new classes of mission architectures. For example, the large shroud diameter will accommodate 8 meter class monolithic primary mirrors, or 15 to 20 meter class segmented primary mirror systems, or even 8 to 16 meter class x-ray telescopes. While a Delta IV has the ability to launch approximate a 4.5 meter diameter payload with a mass of 13,000 $\mathrm{kg}$ to L2, the Ares V is projected to have the ability to launch an 8 meter diameter payload with a mass of 60,000 kg to L2 and $180,000 \mathrm{~kg}$ to Low Earth Orbit. 
Please note that the Ares V vehicle specifications are in a constant state of flux and final performance capabilities will not be known for several years. Most of the performance specifications discussed in this paper are for launch vehicle configuration LV 51.00.39. LV 51.00.39 configuration is a 10 meter diameter 110 meter tall rocket with a core stage and an upper stage. The core stage has five RS-68 engines and two 5-segment solid rocket boosters. The upper stage uses a single J-2X LOX/LH2 engine. An alternative configuration with significantly more performance capability is launch vehicle LV 51.01.48. LV 51.01.48 has a core stage with six RS-68 engines and two 5.5-segment SRBs which provides approximately $180,000 \mathrm{~kg}$ to $\mathrm{LEO}$ and $62,500 \mathrm{~kg}$ to $\mathrm{L} 2$.

\section{ARES-V LAUNCH CAPABILITY}

NASA's Ares V cargo launch vehicle, planed to enter service in 2019, completely changes the paradigm of future space science mission concepts. It has the potential to revolutionize space astronomy by being able to place into orbit far more volume and mass than any existing system (Table 1). [1,2,3,4,5]

\begin{tabular}{|l|l|l|l|l|l|l|l|}
\hline \multicolumn{7}{|c|}{ Table 1: Current and Planned Launch Vehicle Capabilities } \\
\hline & Fairing Dia & Payload Volume & Mass [kg] to & GE & \multicolumn{1}{l|}{} \\
\cline { 4 - 9 } & & & LEO & GTO & GEO & L2 & Escape \\
\hline Space Shuttle & & $4.6 \mathrm{~m} \times 18.3 \mathrm{~m}$ & 25,061 & NA & NA & NA & NA \\
\hline Ariane 5 ECA & $5 \mathrm{~m}$ & $4.5 \mathrm{~m} \times 15.5 \mathrm{~m}$ & 21,000 & 10.500 & & 6,600 & 4,300 \\
\hline Atlas V HLV & $5 \mathrm{~m}$ & $4.5 \mathrm{~m} \times 12.9 \mathrm{~m}$ & 29,420 & 13,000 & 6,350 & & \\
\hline Delta IV H & $5 \mathrm{~m}$ & $4.5 \mathrm{~m} \times 16.3 \mathrm{~m}$ & 22,950 & 12,980 & 6,160 & & 9,305 \\
\hline Ares V (LV 51.00.59) & $10 \mathrm{~m}$ & $8.8 \mathrm{~m} \times 17.2 \mathrm{~m}$ & 143,000 & 70,300 & 36,200 & 55,600 & 53,000 \\
\hline
\end{tabular}

The maximum payload mass which can be delivered to various orbits (SE-L2, GEO, etc.) was analyzed assuming a direct insertion without gravity assist.

The current baseline shroud is a biconic fairing with a $10 \mathrm{~m}$ outer diameter and $21.7 \mathrm{~m}$ height providing an 8.8-m diameter by $17.2-\mathrm{m}$ tall dynamic envelope for a payload volume of 860 cubic meters. This volume is nearly three times that of the Space Shuttle payload bay. A leading contender to replace the biconic is an ogive configuration (Figure 1) which would provide even more payload volume and useable cylindrical vertical height. A longer 'stretch' 10-m fairing has been considered that is 26 meter tall with 1410 cubic meters of volume.

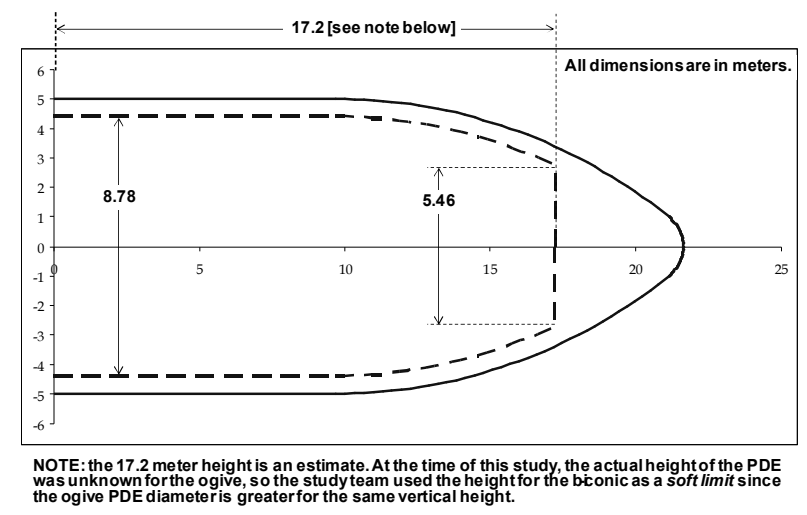

Figure 1 Ogive Faring Dimensions

The predicted Ares V launch environment launch loads (summarized in Table 2) are similar to those for existing launch vehicles. Please note that these loads are not concurrent. They are the maximum load experienced at some time during launch. And, the lateral loads do not include wind loading or vibration.

\begin{tabular}{|l|l|l|}
\hline Table 2 Predicted Maximum Launch Loads of an Ares V \\
\hline Maximum Launch Load & Steady State & Dynamic \\
\hline
\end{tabular}




\begin{tabular}{|l|l|l|}
\hline Axial $(\mathrm{Z})$ & 4 g's & $+/-1$ g's \\
\hline Lateral $(\mathrm{Y})$ & $1.5 \mathrm{~g}$ 's & $+/-0.5 \mathrm{~g}$ 's \\
\hline Acoustic & & $152 \mathrm{~dB}$ \\
\hline
\end{tabular}

The maximum payload mass which can be delivered to Low Earth Orbit (LEO) as a function of altitude and inclination angle was analyzed (Figure 2). The Hubble Space Telescope is at an orbit of $590 \mathrm{~km}$ with an inclination angle of 28.5 deg. The International Space Station is at an orbit of 360 to $440 \mathrm{~km}$ with an inclination angle of $51.6 \mathrm{deg}$. Payload mass includes spacecraft, payload adapter and any mission specific hardware.

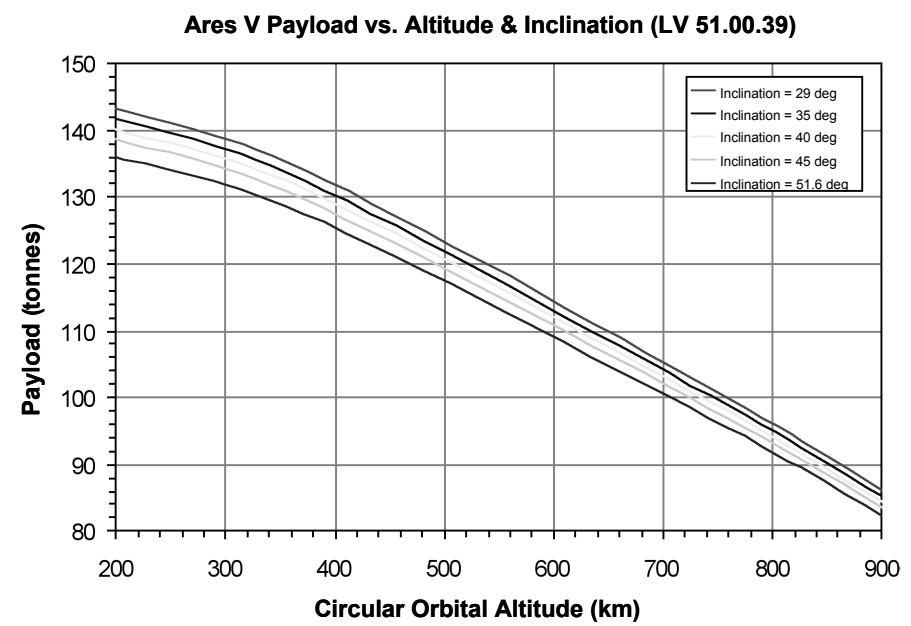

Figure 2: Ares V Payload Mass (metric tons) to Low Earth Orbit depends on Orbit Altitude and Inclination Angle, the higher the orbit or greater the inclination angle, the less mass which can be launched. Data is for LV 51.00.39

\section{POTENTIAL SCIENCE MISSIONS}

For over 30 years, science mission capabilities have been constrained by launch vehicles. The Hubble Space Telescope and Chandra X-ray telescope were specifically designed to match the Space Shuttle's payload volume and mass capacities. And, the James Webb Space Telescope is currently being designed to match the capacities of an Ariane 5. Now, the planned Ares V mass and volume payload capacities could significantly enhance astronomical science by allowing much larger telescopes, which in turn enables the observation and study of much fainter objects. [6,7] Four specific potential astrophysics missions which could be enabled or enhanced by the Ares V include: ATLAST-8, ATLAST-16, SAFIR and Gen-X.

\subsection{ATLAST-8}

ATLAST-8 (Advanced Technology Large Aperture Space Telescope) is an 8-m monolithic UV/Optical telescope (Figure 3). ATLAST-8 would have unprecedented sensitivity and angular resolution in the optical region. It could investigate a wide range of important astronomical topics, such as how the modern Universe formed, how galaxies form, and how planetary systems arise from circumstellar disks. And, it would be able to study the atmospheres of exosolar planets.

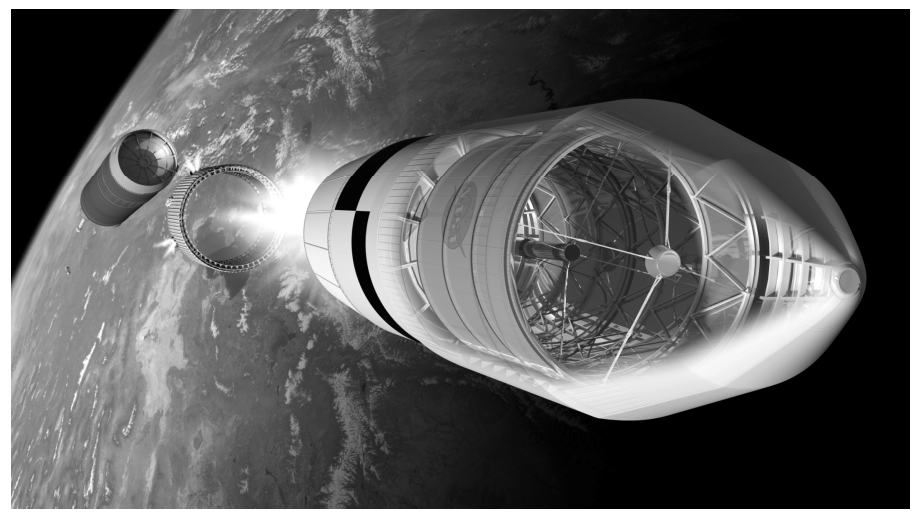

Figure 3 Ares V can launch an 8 meter monolithic mirror telescope. 
ATLAST-8 is specifically designed to fit inside the Ares V 10 meter diameter fairing (Figure 4). The 17.2 meter vertical center height is completely sufficient to allow for a fully deployed telescope to stand up inside the fairing with its primary mirror towards the bottom (with its surface normal parallel to the direction of launch) and its secondary mirror towards the top. The primary mirror structure supports the entire mass of the observatory, i.e. the primary mirror, the optical bench, the spacecraft and the science instrument module. This mass is then transferred through the payload adapter fixture (PAF) to the Ares V outer shell. A Hubble style cylindrical optical bench meters the distance to the secondary mirror spiders. The height of this bench is determined by the cylindrical height of the fairing. Four doors seal the payload package on the ground from potential contamination.

ATLAST-8 is specifically designed to take full advantage of the Ares V's ability to deliver a $65,000 \mathrm{~kg}$ payload to Sun-Earth L2. The total observatory (telescope, science instruments and spacecraft) has an estimated dry mass of $\sim 51,400 \mathrm{~kg}$ with a 45\% margin against the Ares V 65,000 kg capacity (excluding the primary mirror whose mass can only decrease as material is removed). If the Ares $\mathrm{V}$ mass capacity were to decrease, the design mass can be reduced to maintain a minimum mass margin of $30 \%$.

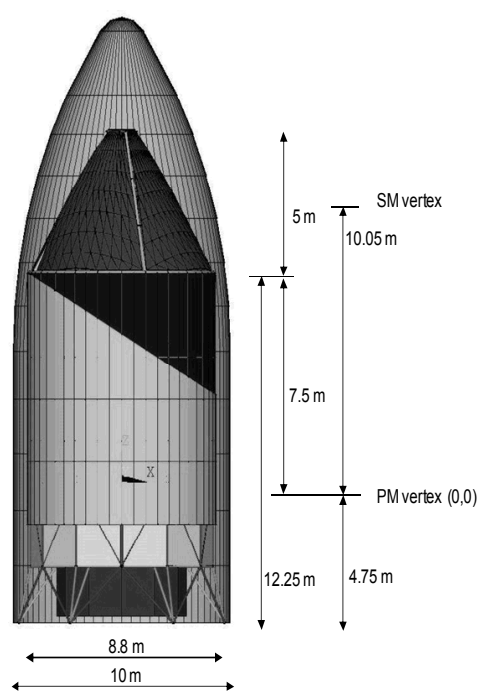

Figure 4 ATLAST-8m Packaged inside Ares V

\subsection{ALTAST-16}

ATLAST-16 is a 16-meter segmented UV/Optical telescope with truly revolutionary capabilities. It could do all the science of ATLAST-8 and more. It would be able to obtain spectra of the atmospheres of Earth-like planets in the habitable zone of thousands of candidate stars. [10]

ATLAST-16 is a JWST class segmented telescope consisting of $36 \sim 2.4 \mathrm{~m}$ (flat-to-flat) hexagonal segments, in a 3-ring arrangement. (Figure 5) The edge-to-edge primary-aperture diameter is $16.8 \mathrm{~m}$. The mass of ATLAST-16 can easily be kept within the Ares V lift capacity using currently available mirror technology. Even if the primary mirror assembly areal density was $50 \mathrm{~kg} / \mathrm{m} 2$, its total mass would be less than half the mass allocated to just the solid glass 8-meter monolithic primary mirror. As with JWST, segment orientation is controlled in six degrees-of-freedom by rigid body actuators.

Packaging the $16.8-\mathrm{m}$ telescope and its instrument suite is a significant challenge, even with the large volume in an Ares $\mathrm{V}$ fairing. Minimizing the primary-secondary mirror distance reduces mass, increases telescope stability, and increases the packaging options. To this end, we constrained the primary mirror focal ratio to 1.5 during the optical design phase. The geometry for the instrument volume depends on the folding scheme used for the telescope. A chord-fold design shares significant heritage with JWST. The primary mirror is folded to fit within the shroud and the secondary mirror is stowed along one edge. This architecture provides a cylindrical stowage volume $16 \mathrm{~m}$ in height $\times 8 \mathrm{~m}$ in width. Within this

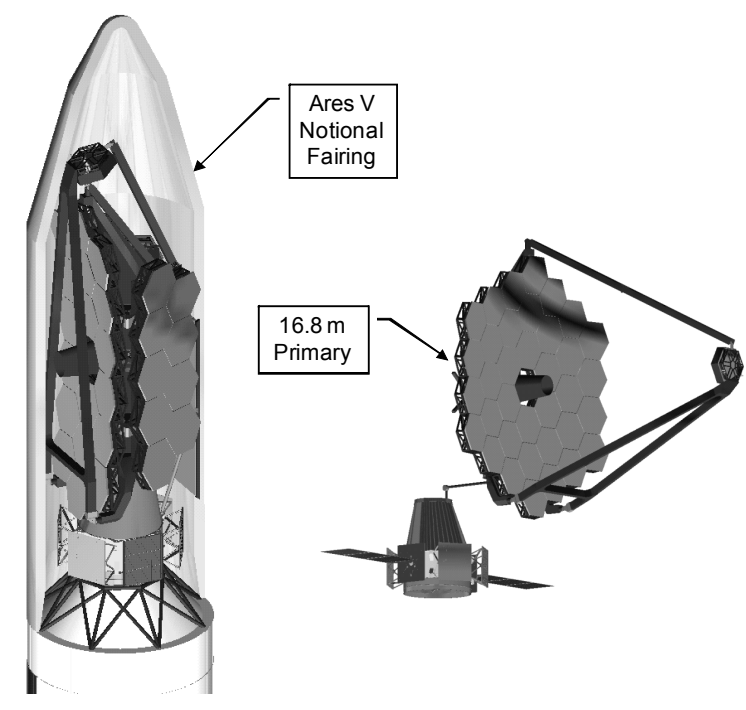

Figure 5. ATLAST-16 stowed in Ares V fairing and deployed (but with sunshade and solar sail, to mitigate solar torque, not shown) volume, a $16 \mathrm{~m} \times 4.5 \mathrm{~m}$ diameter volume is available for instrument packaging. The disadvantages of this configuration are the increased center of gravity offset of the payload and reduced structural stiffness of the metering structure. In addition the secondary mirror must be replaced with a hexapod (vs. JWST's tripod) to achieve reasonable $(>10 \mathrm{~Hz})$ decenter/despace frequencies. 


\subsection{SAFIR}

SAFIR (Single Aperture Far-Infrared) will explore the FIR (20 to 300 micrometer) universe with higher sensitivity and spatial resolution than previously achieved. It will provide new insights into the cosmic history of star formation and nucleosynthesis. Diffraction limited at 20 micrometers, SAFIR needs to operate at temperatures below 10K. [11] Similar to the ATLAST project, there are at least two potential SAFIR mission concepts: monolithic and segmented. The planned Ares V enables the ability to build and launch an 8-meter class monolithic SAFIR, or a 16-meter class segmented SAFIR or even a larger 20 to24 meter class segmented SAFIR [12] (Figure 6). An approximately 20-m diameter telescope would have 10 times the sensitivity and 2-3 times the spatial resolution of JWST. This high sensitivity and spatial resolution would enable SAFIR to both detect and individually distinguish galaxies in a manner that is much less affected by source confusion. In nearby galaxies, it could study star formation, resolve details of planetary system formation, and trace the chemical processes that lead to life.

Because SARIR's diffraction limited wavelength is 40X longer than ATLAST (20 micrometers vs 0.5 micrometers), a SAFIR observatory should be significantly easier and lower cost to manufacture. The mirror quality can be much relaxed as well as the telescope stiffness requirement for system alignment and mechanical stability. Because of these relaxed stiffness requirements, the launch mass may be as low as $10 \mathrm{mT}$ or $\sim 20 \%$ of the launch capacity of Ares V for a mission to Sun-Earth L2. This could allow for significant augmentations to the observatory such as more/larger instruments, as well as an enhanced spacecraft. A large segmented SAFIR should be achievable with existing JWST technology.

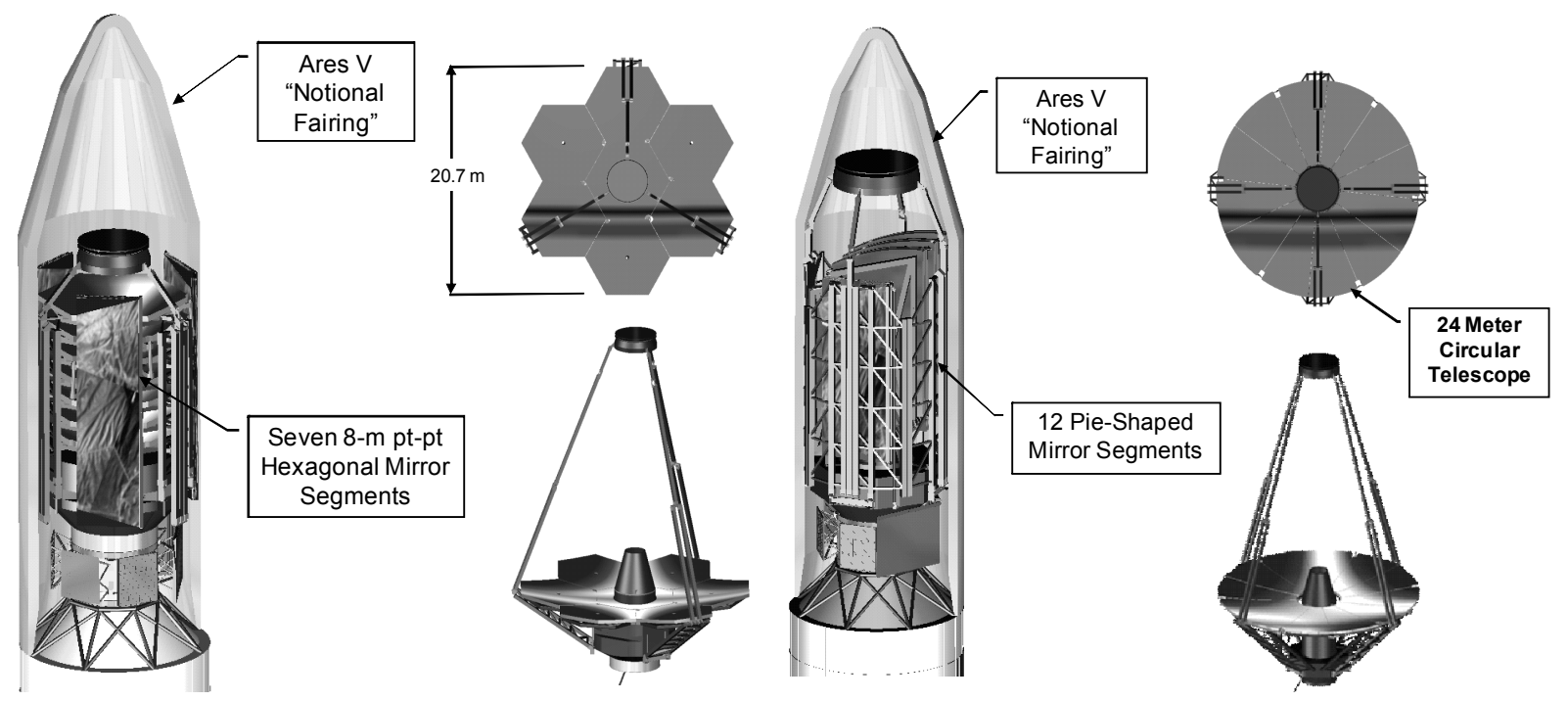

Figure 6. Concepts for packaging in an Ares V fairing and deploying a 20 meter class segmented telescope using 8 meter point to point hexagonal mirror segment or a 24 meter class segmented telescope with a circular aperture using pie segments. 


\subsection{Generation-X}

Generation-X proposes to study the early universe when the first black holes, stars, and galaxies formed, as well as their evolution with cosmic time. Gen-X would also provide new insights into the physics of matter in extreme environments. [13]

Key requirement necessary to enable Gen-X science are that the effective area at $1 \mathrm{keV}$ is $50 \mathrm{~m} 2$ and the angular resolution is $0.1 \mathrm{arcsec}$. To meet the angular resolution requirement requires a mirror with a diameter of at least 12 meters. To meet the effective area requirement of $50 \mathrm{~m} 2$ requires about $104 \mathrm{~m} 2$ of total glass area. This is accomplished with 40 to $50 \mathrm{~cm}$ long mirror segments stacked $\sim 0.1-0.2 \mathrm{~mm}$ apart. While such a mirror is very massive, it is well within the Ares V capacity. The Ares V enables a simplified and cost-effective mission concept which can be deployed in a single launch. The baseline concept folds a partially filled 16-m diameter mirror into the Ares V 10-m fairing. The X-ray telescope is delivered directly to SunEarth L2. The estimated spacecraft mass of $22 \mathrm{mT}$ is far less than the $65 \mathrm{mT}$ Ares V capability. Since the telescope is volume-limited, not mass limited, the mass margin enables design freedom for the optics, structure, supporting

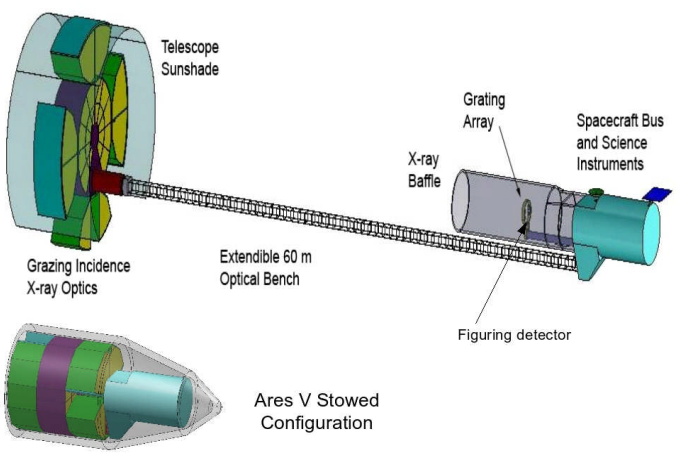

Figure 7 Gen-X Stowed in Ares V Fairing and Deployed. electronics, and the science instruments.

\section{CONCLUSION}

NASA's planned Ares V cargo launch vehicle's payload mass and volume capabilities completely change the paradigm of future astrophysics and planetary space science mission architectures. Its ability to place 140,000 to $180,000 \mathrm{~kg}$ of payload into Low Earth Orbit and $\sim 60,000 \mathrm{~kg}$ of payload into Sun-Earth L2 inside of a 10 meter diameter fairing enables entirely new generations of space telescopes. Potential space telescopes enabled by an Ares V include: an 8 meter class monolithic primary mirror ultraviolet/visible observatory; a 16 meter class segmented UV/optical telescope; a 16 to 24 meter class segmented far-infrared telescope; and a 12 to 16 meter class x-ray observatory. These next generation great observatories for the $21^{\text {st }}$ century offer very high angular resolution, very high sensitivity, broad spectral coverage, and high performance stability. But of equal importance is that they do not require any new technology. Because of the Ares V's mass and volume capacities, these observatories can be built today with existing technology. Mission designers can use simple mature technology to eliminate complexity and reduce program cost and increase mission reliability as well as science return. Thus, allowing NASA to invest its time and resources to mature technology to reduce risk and enhance performance. And, to start investing in developing technology to enable even more advanced mission concepts such as synthetic aperture observatories via formation flying or 100 meter class apertures via on-orbit assembly. 


\section{REFERENCES}

1. Arianespace, Ariane 5 User's Manual Issue 4, 2004

2. United Launch Alliance, Atlas Product Sheet, 2006

3. United Launch Alliance, Delta Product Sheet, 2006

4. International Launch Services, Atlas Launch System Mission Planner's Guide, 1999

5. Boeing, Delta IV Payload Planners Guide, 2000

6. Langhoff, Stephanie, Dan Lester, Harley Thronson, Randy Correll, "Workshop Report on Astronomy Enabled by Ares V”, NASA/CP-20080215361, April 26-27, 2008

7. Thronson, Harley, Daniel F. Lester, Stephanie R. Langhoff, Randall R. Correll, and H. Philip Stahl, “Ares V and Future Very Large Launch Vehicles to Enable Major Astronomical Missions", $59^{\text {th }}$ International Astronautical Congress, Glasgow, Scotland, Sept 2008.

8. Stahl, H. Philip, "Design study of an 8 meter monolithic mirror uv/optical space telescope", SPIE Astronomy Conference, Marseille, France, June 2008

9. Stahl, H. Philip and Marc Postman, "8 meter Monolithic UV/Optical Space Telescope", 59 ${ }^{\text {th }}$ International Astronautical Congress, Glasgow, Scotland, Sept 2008.

10. Postman, Marc, et. al. "Advanced Technology Large-Aperture Space Telescope (ATLAST): A Technology Roadmap for the Next Decade”, Final Report for NASA’s Astrophysics Strategic Mission Concept Study, 24 April 09.

11. Lester, Dan, et. al., Large Infrared Telescopes in the Exploration Era - SAFIR, SPIE Proc.6687, (2007).

12. Lilly, Chuck, "Future Deployment Systems and Very Large Fairings", Ares V Astronomy Workshop, ARC, NASA, 27 April 2008.

13. Brissenden, Roger, "Generation-X: A Mission Enabled by Ares V”, Ares V Astronomy Workshop, ARC, NASA, 27 April 2008. 